\title{
Blind spot size depends on the optic disc topography: a study using SLO controlled scotometry and the Heidelberg retina tomograph
}

Jörg Heinrich Meyer, Mechthild Guhlmann, Jens Funk

\begin{abstract}
Aims-To find out whether the size of the blind spot area, determined by static perimetry, depends on the surface topography of the optic disc and its surrounding area.

Methods-Ten eyes were examined; all had a parapapillary atrophy adjacent to the temporal side of the disc. Microperimetry was performed under direct fundus control using a Rodenstock scanning laser ophthalmoscope. The horizontal meridian of the optic discs was examined in $0.5^{\circ}$ steps using five stimulus sizes (Goldmann I to $\mathrm{V}$ ), each with 10 different degrees of brightness. Optic disc topography was measured with the Heidelberg retina tomograph (HRT).

Results-Stimuli with a high luminance level (Goldmann IV, $4 \mathrm{~dB}$ ), presented on the horizontal meridian, were seen up to $0.75^{\circ}$ centrally (that is, towards the optic disc centre) from the temporal edge of the parapapillary atrophy but up to $1.85^{\circ}$ centrally from the nasal optic disc border $(\mathbf{p}<0.01)$. Horizontal HRT section profiles of the optic disc consistently showed prominent nasal disc borders contrasting with a shallow excavation within the temporal parapapillary atrophy.

Conclusions-The size of scotomas depends on the surface topography of the tested area. The prominent nasal part of the optic disc appears less 'blind' than the shallow temporal part, probably because of more intensive light scattering by the prominent nasal part of the disc. These considerations should also apply to other scotomas.
\end{abstract}

(Br f Ophthalmol 1997;81:355-359)

It is known that a scotoma (for example, the blind spot) can decrease in size when the luminance level of the test stimuli increases. Von Helmholtz (quoted in Le Grand ${ }^{1}$ ) was probably the first to describe the phenomenon of a diffuse flash, which is noticed by many individuals, when stimuli with a high luminance level are projected into the centre of the optic disc, even if the stimulus size is much smaller than the disc diameter. Fankhauser and Haeberlin, using a cupula perimeter, showed that this effect was due to entoptic light scattering and not instrumental stray light. ${ }^{2}$ Furthermore, they published quantitative results concerning stray light emitting properties of the disc. ${ }^{2}$ When publishing those results, however, the stimuli could not be set under direct fundus control. Thus, correlation of the blind spot borders with the anatomic margins of the optic disc was not possible. Today, this can be achieved with the scanning laser ophthalmoscope (SLO). ${ }^{34}$ Our group has already determined threshold characteristics for normal optic discs using SLO controlled static perimetry. ${ }^{5}$ One of our test candidates was a moderately myopic woman with a shallow temporal parapapillary atrophy, whose blind spot border shifted much more towards the disc centre at the nasal side than at the temporal side, when the luminance level of the stimuli was increased. This raised the question of whether the size of the 'blind spot' area depends on the surface topography of the optic disc and its surrounding area.

\section{Methods}

We examined 10 eyes of five healthy, moderately myopic volunteers (age 30 (SD 4) years, refraction -2.0 to $-6 \mathrm{D}$, three males and two females), who all had a shallow parapapillary atrophy adjacent to the temporal side of the disc and fairly prominent nasal disc borders. Demographic data are summarised in Table 1.

Visual acuity was $\geq 10 / 10$ and slit-lamp biomicroscopy, as well as indirect ophthalmoscopy, did not reveal any abnormalities. Thirty degree photographs of the disc and the parapapillary fundus were taken and used as a reference for the determination of the nasal and temporal disc borders. Standard light sense perimetry (Octopus, G1 program) was normal in all cases.

Our study followed the tenets of the Declaration of Helsinki and informed consent was obtained before the examinations.

Microperimetry was performed within a $32^{\circ}$ $\times 24^{\circ}$ field of the central fundus under direct fundus control using a Rodenstock scanning laser ophthalmoscope (scotometry program,

Table 1 Demographic and refractive data

\begin{tabular}{lllllll}
\hline & Patient & Age & Sex & Eye & Visual acuity & Refraction \\
\hline 1 & MG & 30 & F & R & $12 / 10$ & -4.75 \\
& & & & L & $12 / 10$ & -6.0 \\
2 & HS & 26 & F & R & $12 / 10$ & -4.0 \\
& & & & L & $12 / 10$ & -3.75 \\
3 & TW & \multirow{2}{*}{32} & M & R & $10 / 10$ & -3.75 \\
& & & & L & $10 / 10$ & -2.0 \\
4 & TE & 27 & M & R & $10 / 10$ & -6.0 \\
& & & & L & $10 / 10$ & -6.0 \\
5 & SH & \multirow{2}{*}{36} & M & R & $10 / 10$ & -3.5 \\
& & & & L & $10 / 10$ & -2.25 \\
\hline
\end{tabular}

Accepted for publication 11 December 1996
Universitäts-

Freiburg, German

M Guhlmann

Correspondence to:

Dr J H Meyer,

Universitäts-Augenklinik

Killianstrasse 5, 79106 


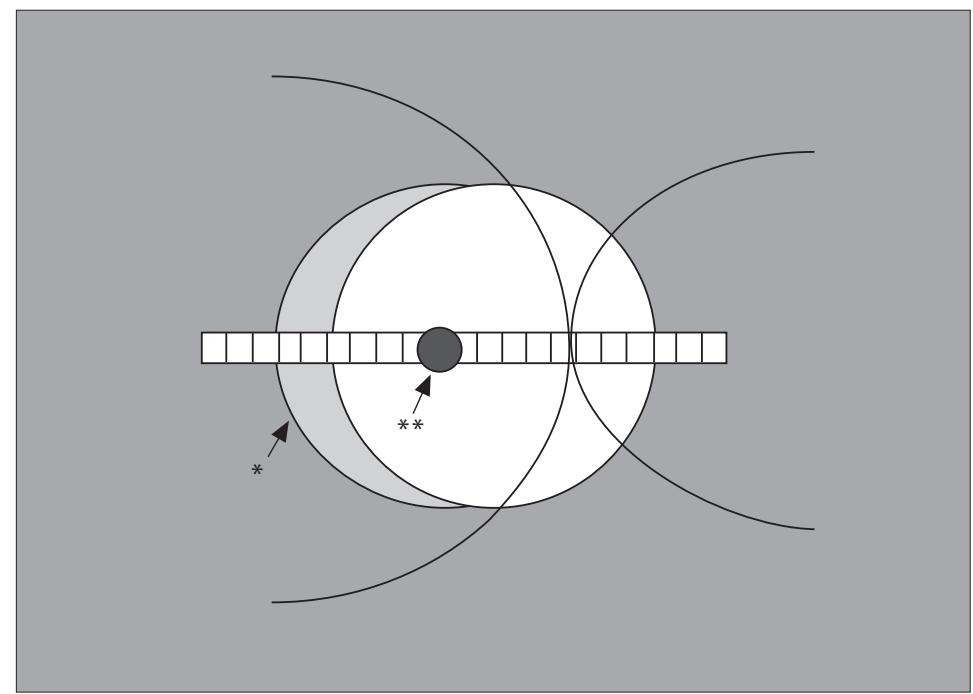

Figure 1 Test stimuli were projected on the fundus using a test grid (row of squares), which is superimposed on the fundus image displayed on the SLO monitor. The centre of the test stimulus is located in the centre of the square. All squares are $0.5^{\circ}$ wide. ${ }^{\star} M a r g i n$ of the temporal crescent, ${ }^{\star \star}$ Goldmann IV, 4 dB stimulus.

version 1.51). Background and stimuli were generated by a helium-neon laser $(633 \mathrm{~nm})$. Background luminance was $6 \mathrm{~cd} / \mathrm{m}^{2}$. Stimulus duration was 0.2 seconds and the brightness could be varied in $1 \mathrm{~dB}$ steps within a range equivalent to 0.2 to $33 \mu \mathrm{W}$. Fundus imaging was achieved by an infrared $(780 \mathrm{~nm})$ diode laser scanning synchronously with the heliumneon laser.

Eye movements cannot yet be corrected with automated routines. ${ }^{6}$ To guide the placement of the test stimuli we have manually superimposed a test grid, printed on a translucent plastic sheet, on the fundus image displayed on the SLO monitor. The temporal edge of the chorioretinal atrophy was aligned with the grid border (Fig 1). Each square corresponded to $0.5^{\circ}$.

We examined the horizontal meridian of the optic discs in $0.5^{\circ}$ steps beginning and ending $1.5^{\circ}$ away from both optic disc margins, using five stimulus sizes (Goldmann I to V), each with 10 degrees of brightness $(0-18 \mathrm{~dB}, 2 \mathrm{~dB}$ steps). About $30 \%$ of all answers had to be neglected because of fixation shifts. Each test point was examined 10 times. If less than $50 \%$ of these 10 stimuli were seen, the test area was considered 'blind'.

As test time was about 4 hours per eye and concentration and, therefore, fixation stability markedly decreased after 30 minutes, we divided the whole examination into 30 minute sections on different days. All volunteers had 15 minutes of training before the first test was started.

The results from the right eye of patient 1 are shown in Figure 2. Confirming former observations (DOG Guhlmann, personal communication) we found that with increasing luminance power (size $\times$ luminance) the border of the blind spot shifted towards the optic disc centre much more at the nasal side than at the temporal side of the disc. This nasotemporal asymmetry was especially pronounced for stimulus IV. Thus we only used this stimulus
$(0-18 \mathrm{~dB})$ in the remaining four patients. The Wilcoxon test was used for statistical comparison.

Small and bright stimuli (Goldmann I, $0 \mathrm{~dB}$ ) were used as well in all patients to determine the sensitivity just at the edge of the discs.

To correlate the perimetric results with the topography we obtained three dimensional $10^{\circ}$ images of the optic disc surface using the Heidelberg retina tomograph (HRT, version $\left.1.11^{7}\right)$.

Using these images we determined depth profiles exactly along the horizontal meridian which we had examined earlier with the SLO.

\section{Results}

The whole area of the optic disc and the parapapillary atrophy turned out to be blind when tested with a bright small stimulus (Goldmann I, $0 \mathrm{~dB}$ ), which was seen everywhere on the fundus (Fig 2). Differential light thresholds were the same just outside the clinically visible border of the blind spot at the nasal and the temporal side.

Larger stimuli were seen within the optic disc area, the largest and brightest stimuli (Goldmann V, $0 \mathrm{~dB}$ ) were seen evenly within the disc (Fig 2).

Stimuli with medium luminance level (Goldmann IV, $4 \mathrm{~dB}$ ), presented on the horizontal meridian of the 10 examined optic discs, were seen on average up to $0.75^{\circ}$ (SD $0.27^{\circ}$ ) centrally (that is, towards the optic disc centre) from the temporal edge of the parapapillary atrophy and up to $1.85^{\circ}\left(0.71^{\circ}\right)$ centrally from the nasal optic disc border $(\mathrm{p}<0.01$, Wilcoxon test). The results of 10 eyes are summarised in Figure 3.

Figure 4 demonstrates the fundus photograph, the HRT intensity image with both the horizontal section profile and the superimposed perimetric result, and the $3 \mathrm{D}$ disc surface reconstruction of the right disc of one test person (No 2).

All 10 patients showed in the horizontal HRT section profiles of the optic disc consistently prominent nasal disc borders contrasting with a shallow excavation within the temporal parapapillary atrophy. In fact, the transition zone between the parapapillary atrophy and the adjacent normal retina was rather steep.

Even though there was some interindividual variation of the nasotemporal asymmetry, the tendency was the same in all cases: within the excavation there was definite 'blindness', as long as the temporal part of the stimulus was within that excavation (that is, the parapapillary crescent), whereas stimuli presented within the prominent area of the disc were seen much more centrally.

\section{Discussion}

Diminution of the blind spot with increasing luminance level is a known and disturbing effect in light sense perimetry. ${ }^{8}{ }^{9}$ According to the data presented here and the data we obtained with normal emmetropes ${ }^{5}{ }^{10}$ the true borders of the scotoma will only be determined correctly with stimuli of an intermediate lumi- 

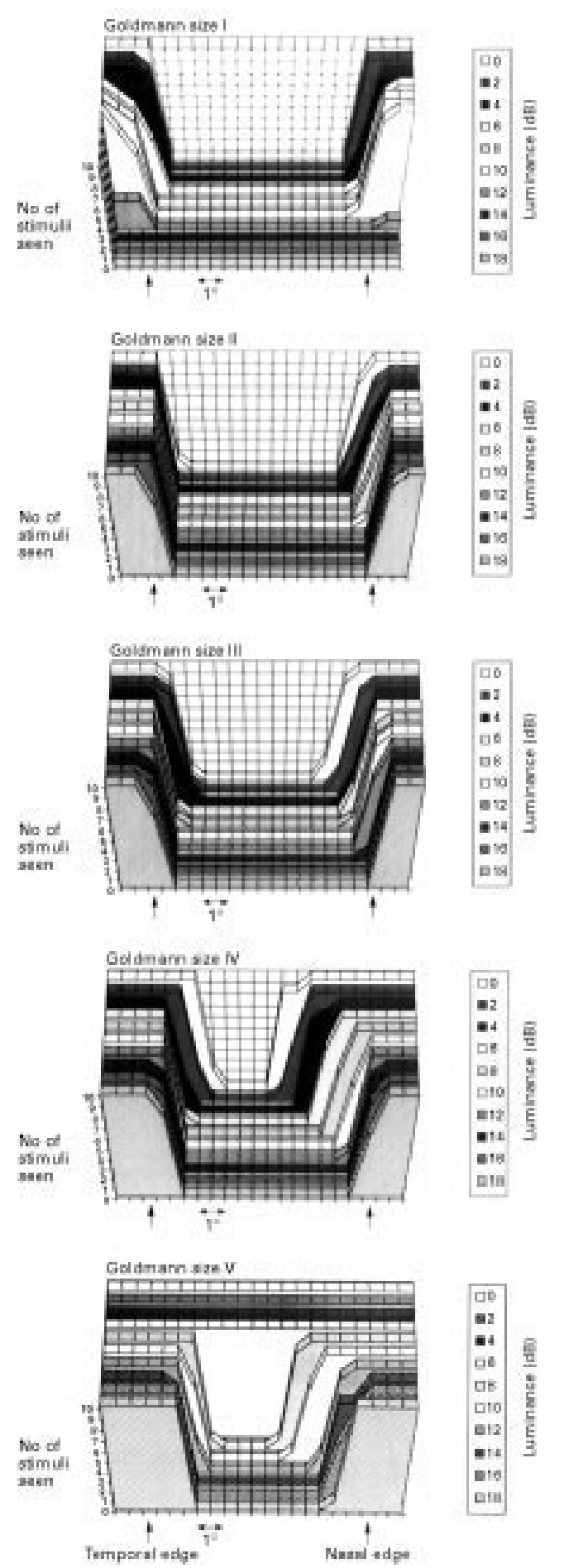

Figure 2 The number of stimuli seen by the test candidate (No 1, right eye, $y$ axis) depends on the test location ( $x$ axis), the luminance ( $z$ axis), and the size of the stimulus. Note that with increasing luminance the border of the blind spot shifts towards the optic disc centre. This effect shows a nasotemporal asymmetry. The left arrows indicate the temporal margin of the parapapillary atrophy, the right arrows the nasal margin of the optic disc.

nance level - that is, Goldmann I, $0 \mathrm{~dB}$ (SLO). With greater luminance level, the scotoma size will be underestimated.

With ordinary automated or kinetic perimetry the 'true' depth and size of scotomas as large as (or smaller than) the blind spot cannot be determined exactly ${ }^{11}$ because of microsac- cades, which exclude exact and consistent stimulus setting. SLO perimetry is advantageous in this regard as it allows fundus control during the examination.

As far as we know, this paper shows for the first time that the size of a scotoma depends on the surface topography of the tested area. The prominent nasal part of the disc appears less 'blind' than the shallow temporal part of the disc (including the parapapillary atrophy), probably due to higher light scattering by the prominent nasal part.

The 'topography effect' did not occur with small (Goldmann I, $0 \mathrm{~dB}$ ) stimuli; they should be used in SLO microperimetry of small retinal lesions.

We believe that these considerations should also apply to other scotomas.

Some questions may arise regarding this topography effect.

(1) Where are the stimuli actually perceived? Areas of the retina with an underlying chorioretinal atrophy do not contain functioning photoreceptors or their amount is heavily reduced, ${ }^{12}$ nor do photoreceptors exist within the (nasal parts of the) optic discs. ${ }^{13}$ Remaining photoreceptors within the parapapillary atrophy (crescent) obviously do not function well because we recorded sharp scotoma borders with small bright stimuli exactly at the temporal edge of the crescent in all 10 cases.

This corresponds with observations of Jonas et $a l^{14}$ and Stürmer et $a l,{ }^{4}$ who found an absolute scotoma within zone $\beta$ (that is, the parapapillary chorioretinal atrophy), the latter using direct laser scanning ophthalmoscopy.

We therefore feel sure that the stimuli presented within the disc were actually 'seen' outside the disc/crescent borders.

\section{(2) Could stray light caused by other structures} contribute to the effect?

It was shown by Miller and Benedek ${ }^{15}$ that the cornea contributes $25 \%$, the lens $50 \%$, and the retina $25 \%$ to the total entoptic stray light.

Because the stimuli were seen better when presented at the (more prominent) nasal parts of the disc in all 10 eyes, this effect should be attributed to retinal (disc surface) light scattering rather than light scattering within the optic media. There is no reason why light scattering within the optic media should favour the retina located nasally to the optic disc.

We have no doubt, however, that the perception of large stimuli presented centrally within the disc area is also caused by scattering of the stimulus light when passing through the optic media of the eye. A sharply delimited stimulus is blurred such that a surrounding halo appears at the retinal surface, which depends on its size and intensity. When presented on a normal functioning retina this halo will be eliminated by lateral inhibition. When presented within the optic disc or another receptor-free area this halo will be perceived by the adjacent normal retina.

This effect may explain why very bright stimuli, presented entirely within the excavated 


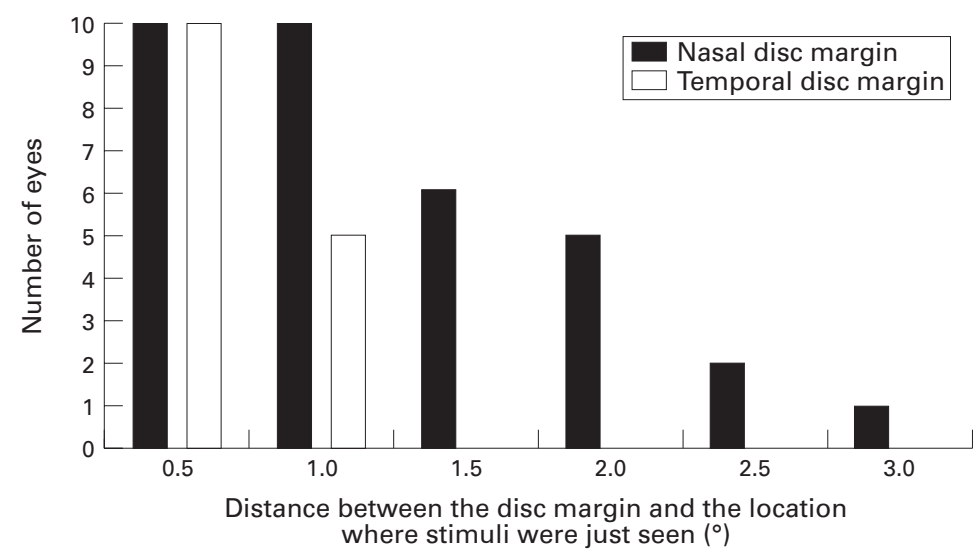

Figure 3 Number of eyes perceiving Goldmann IV, 4 dB stimuli depending on the distance of the disc margins to the centre of the stimulus. Note the marked difference between the nasal and the temporal disc side.

temporal parapapillary atrophy can be seen at all (Fig 2).

(3) Is the retina adjacent to the optic disc more sensitive at the nasal side?

Differential light sensitivity thresholds are lower at the temporal side of the optic disc than at the nasal side. ${ }^{11}$ Using microperimetry, we found, that the differential light sensitivity of the area directly adjacent to the optic disc was almost the same at both horizontal borders in test volunteers without a crescent $t^{5}$ as well as in the eyes presented here.
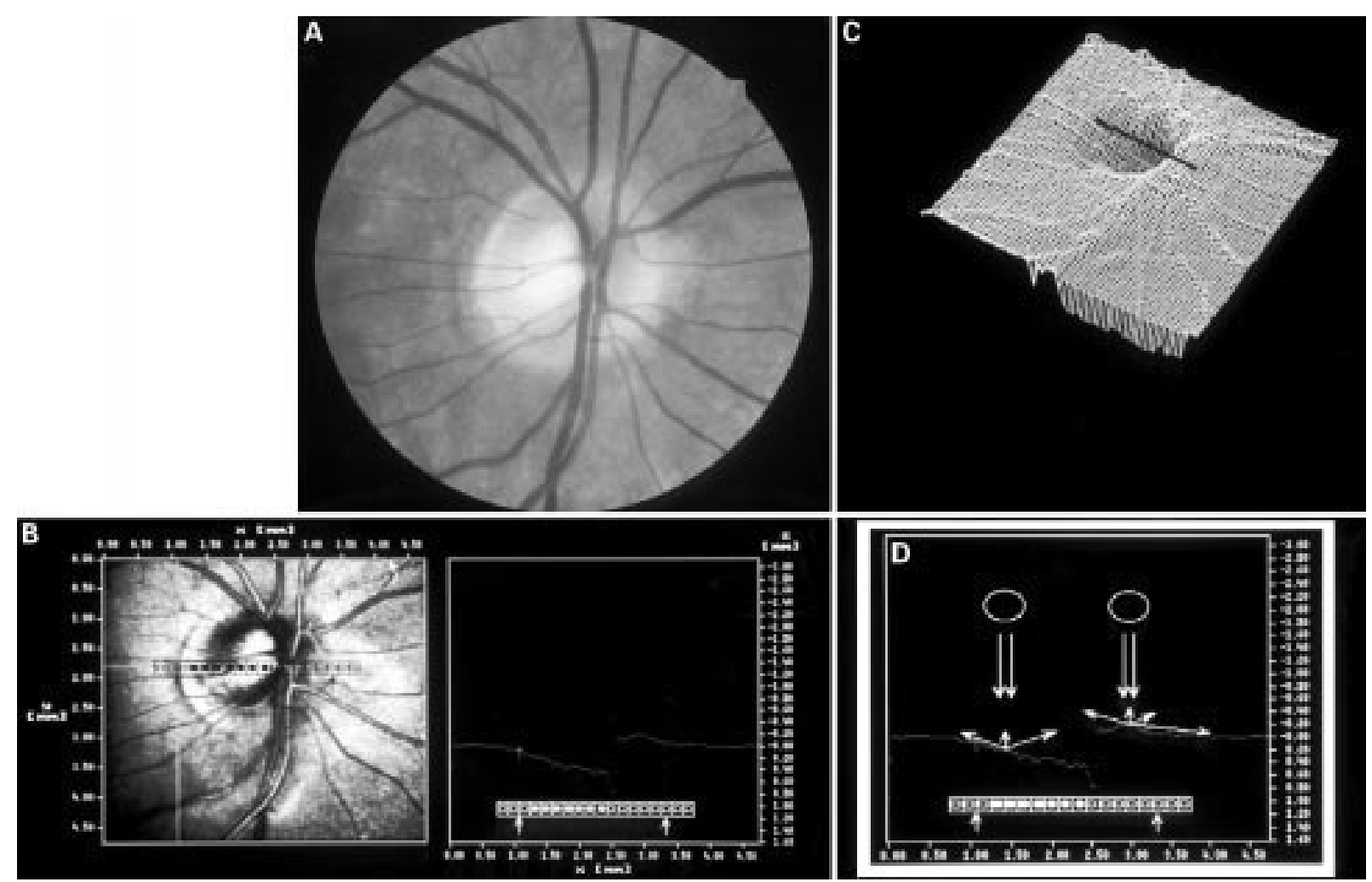

Figure 4 Results of patient No 2, right eye: (A) $30^{\circ}$ fundus photograph. (B) HRT intensity image with the overlaid perimetric result using Goldmann stimulus IV, $4 d B$ (open circles, seen; closed circles, not seen) and the horizontal section profile measured along the horizontal line. (C) 3 D disc surface reconstruction. Line shows the meridian, which the test stimuli were projected on. (D) Possible scattering of the incoming light (long white arrows) by the fundus (optic disc) surface. Note that stray light striking the optic disc/crescent within the excavation should not reach the adjacent functioning retina directly, while stray light originating from prominent nasal parts of the disc should be able to do that. The lower white arrows show the temporal margin of the parapapillary atrophy (left) and the nasal margin of the optic disc (right). 
We expect, for example, that the growth of intraocular tumours may be underestimated when they are examined with perimetry (stimulus size $\geq$ Goldmann II) during follow up; if a tumour, which is flat in the beginning of the disease, becomes more and more prominent the topography effect may mask the true increase of the scotoma.

The opposite may occur if the (retinal) surface becomes more excavated. According to the data presented here one may expect that cupping of the optic disc in early glaucoma, which, as we know, precedes visual field loss, may lead to less light scattering by the (excavated) disc surface towards the adjacent retina and thereby leads to a 'baring' (enlargement) of the blind spot. This 'baring' has been reported in early glaucoma by some authors ${ }^{16}{ }^{17}$ while others did not find it, ${ }^{18}$ possibly because the patients had different disc topographies or the luminance level of the test stimuli was different.

In summary, we found that the size of the blind spot not only depends on the receptive function of the tested retina and on the luminance power of the test stimulus but also on the surface topography of the tested area. The prominent nasal part of the disc appeared less 'blind' than the shallow temporal part of the disc (including the parapapillary atrophy), probably as a result of higher light scattering by the prominent nasal part of the disc.

As the 'topography effect' did not occur with Goldmann I, $0 \mathrm{~dB}$ stimuli, these should be used in SLO microperimetry of small retinal lesions.

These considerations should also apply to other scotomas.
Preliminary data were presented at the ARVO annual meeting, May 1995, Ft Lauderdale, Florida, USA.

1 Le Grand Y. Recherches sur la diffusion de la lumière dans l'oeil humain. Rev Opt Theor Instrum 1937;8:241-66.

2 Fankhauser F, Haeberlin $\mathrm{H}$. An estimation of the falsifying effect of stray light in perimetry. Doc Ophthalmol 1980;50: effect of

3 Webb RH, Hughes GW, Delori FC. Confocal scanning laser ophthalmoscope. Appl Optics 1987; 26:1492-9.

4 Stürmer J, Schroedel C, Rappl W. Low-backgroundbrightness static SLO fundus-perimetry Invest Ophthalmol Vis Sci 1990;31:504.

5 Guhlmann M, Meyer JH, Funk J. SLO-controlled fundus nance power of the test stimuli. Ger 7 Ophthalmol (Suppl). 1995;4:93.

6 Becker M, Rohrschneider K, Schumacher N, Fendrich T, Kruse FE, Völcker HE. Scanning laser ophthalmoscope (SLO): full-threshold static perimetry-sensitivity values and reproducibility in normals. Invest Ophthalmol Vis Sci
1995;36:238.

7 Zinser G. Topographische Messungen am Augenhintergrund mit dem Heidelberg Retina Tomograph. In: Kampik A, ed. Fahrbuch der Augenheilkunde 1992, Laser. Zülpich: A, ed. Fahrbuch der Augenh

8 Bek T, Lund-Andersen H. The influence of stimulus size on perimetric detection of small scotomata. Graefes Arch Clin Exp Ophthalmol 1989;227:531-4.

9 Stepanik J. Der blinde Fleck: Kritische Betrachtung rasterperimetrischer Aussage über ein Skotom bekannter Größe. Klin Monatsbl Augenheilkd 1986;189:409-12.

10 Meyer JH, Guhlmann M, Funk J. SLO-controlled static fundus perimetry: blind spot size depends on optic disc topography (HRT). Invest Ophthalmol Vis Sci 1995;36:454.

11 Flammer J, Jener F, Bebie H, Keller B. The Octopus glaucoma G1 program. Glaucoma 1987;9:67-72.

12 Jonas JB, Königsreuther KA, Naumann GOH. Histomorphometry of the parapapillary region in glaucomatous and normal human eyes. Invest Ophthalmol Vis Sci 1990;31:456.

13 Glaser S. Anatomy of the visual sensory system. In: Tasman W, Jaeger EA, eds. Duane's clinical ophthalmology. Philadelphia: Lippincott, 1992:2(4), 1-2.

14 Jonas JB, Gusek GC, Fernández MC. Correlation of the blind spot size to the area of the optic disc and blind spot size to the area of the optic disc and

15 Marapapillary atrophy. Am f Ophthalmol 1991;111:559-65. IL: Thomas, 1973:2, 32.

16 Tranquair HM. Glaucoma with special reference to medical aspect and early diagnosis. BMF 1935;3906:922.

17 Gramer E, Gerlach R, Krieglstein GK, Leydhecker W. Zur Topographie früher glaukomatöser Gesichtsfeldausfälle bei der Computerperimetrie. Klin Monatsbl Augenheilkd 1982; 180:515.

18 Drance SM. The early field defects in glaucoma. Invest $O p h-$ thalmol Vis Sci 1969;8:84. 